\title{
Utilizing Community Promotoras to Increase Cervical Cancer Screening in Hispanic Women
}

\section{Heard C* \\ The University of Texas Medical Branch, USA}

*Corresponding author: Connie Heard, The University of Texas Medical Branch, 301

University Blvd Galveston Texas 77555, USA, Tel: 817-907-4541; Email:

coheard@utmb.edu

\section{Research Article \\ Volume 2 Issue 2}

Received Date: March 20, 2018

Published Date: April 09, 2018

\section{Abstract}

Objectives: To increase the rate of kept appointments for cervical cancer screening in Hispanic women from a nurse managed clinic.

Methods: A retrospective electronic chart review was conducted to identify the subjects for the two groups of Hispanic women eligible for cervical cancer screening. The control group received the usual standard of care for cervical cancer screening education by advanced practice nurses(APNs) and the intervention group received a one-hour educational class by the trained community promotora. Following the educational class, intervention group participants were able to schedule an appointment for Pap/HPV testing.

Results: There is no statistically significant difference in the rate of kept appointments between the control group and intervention group.

Conclusion: Hispanic women educated by trained community promotoras about cervical cancer prevention and screening does not prove to be more effective in increasing rates of kept appointments at a nurse managed clinic; compared to the usual standard of care with the APNs educating patients on cervical cancer prevention and screening.

Keywords: Promotora; Cervical cancer prevention; Cervical cancer screening; Pap testing; Hpv testing; Cancer disparities

Abbreviations: APNs: Advanced Practice Nurses; WHO: World Health Organization; CDC: Centers for Disease Control and Prevention; HPV: Human Papilloma Virus; CHWs: Community Health Workers; APNs: Advanced Practice Nurses; ACS: American Cancer Society; NCI: National Cancer Institute; IRB: Institutional Review Board; USPSTF: U.S. Preventative Services Task Force.

\section{Introduction}

In 2014, the United States estimated 12,360 women who were diagnosed with cervical cancer, while an estimated 4,020 deaths have been linked to cervical cancer [1]. From 2004-2010, the lifetime risk of cervical cancer in the United States is 0.7 per 100,000 based on 2009-2011 data with a five-year survival rate of $67.9 \%$ 


\section{Nursing \& Healthcare International Journal}

[1]. Although, the number of cervical cancer cases has significantly declined over the past several decades due to Pap testing, women continue to die of cervical cancer in the United States (Centers for Disease Control and Prevention) [2]. The estimated annual cost per person aged 65 or older for cervical cancer care in the United States is $\$ 45,174$ initially, with $\$ 1,425$ for continued care excluding the last year of life care, which can be as high as $\$ 78,553$ [3]. Based on the statistical data presented, further interventions are needed to prevent cervical cancer. One of the most substantial risk factors for cervical cancer is the human papilloma virus (HPV). The HPV is considered a sexually transmitted infection commonly known as genital warts.HPV is responsible for up to $20 \%$ of the deaths related to cervical cancer [4]. Of the women in the United States diagnosed with cervical cancer, $90 \%$ of them have been exposed to HPV. Virus type 16 and type 18 cause approximately two thirds of all cervical cancer [4]. HPV vaccinations, Cervarix and Gardasil, are effective in combating the HPV viral infection. These vaccinations are available for boys, girls and young adults before they become sexually active [2]. Although vaccinations are available, lack of knowledge regarding these vaccinations, lack of knowledge regarding the disease, and the inability to pay for vaccinations may be a barrier to the use of the HPV vaccination [2].

Race and ethnicity are important factors in the rate of cervical cancer diagnosis and mortality. African Americans and Hispanics have a higher incidence of cervical cancer than any other ethnicity. This statistic is most likely related to a decrease in access and resources for Pap testing and treatment [2]. According to Healthy People 2020, the definition of health disparity is "a particular type of health difference that is closely linked with social, economic, and/or environmental disadvantages" [5]. Socioeconomic status is a major influence on the underlying risk factors for cancer. For example, the poorer the person is the greater the risk to be a smoker and less likely to seek medical care when needed. In comparison to African Americans and Caucasians, Hispanic women have the highest percentage of women who are living below poverty and that are underinsured [6]. Cancer disparities in accordance to race and ethnicity exist because of the varying social classes which are defined by educational level. The higher the level of education, the greater decline in cancer mortality [7]. In 2011, Hispanic women had the highest incidence of cervical cancer in the United States with a rate of 9.8 women per 100,000 population. Black women had the highest rate of death related to cervical cancer [2]. The incidence of comorbidities is much higher in Hispanic women from a lower socioeconomic status, contributing to negative health outcomes with cervical cancer treatment [2].

A major barrier to cervical cancer screening for Hispanic women is the fear of cancer "fatalism" which can decrease screening services in Hispanic women [4]. The fatalistic beliefs of Hispanic women is that they perceive they are unable to prevent cancer, and if they are diagnosed with cancer then the outcome would be death. Hispanic women feel strongly about cancer fatalism than non-Hispanic women [8].

Research studies have shown the positive impact of educational and social support in alleviating fears associated with cervical cancer screening. For example, one published study conducted in Chiapas, Mexico revealed that the use of trained community health workers (CHWs), also known as "promotoras" which are laypersons who are familiar with the Hispanic community and culture can improve cervical cancer screening on high-risk Hispanic women ages 30 to 55 years old [9]. The two groups in the study were one rural traditional and one rural promotora group. The rural promotora group were recruited by trained community health workers (CHWs) from the surrounding location and the traditional group was self-referred for cervical cancer screening. The screening event allowed the participants to complete a questionnaire that asked questions related to knowledge, beliefs, and social support which included questions on transportation and childcare services to attend the cervical cancer screening event. The findings of the study revealed that the group of Hispanic women recruited and given cervical cancer education and social support by the CHWs also known as promotoras answered more agreeably to questions related to knowledge and beliefs about cervical cancer and screening. Issues related to "fatalism" were included on the questionnaire and a significant number of women recruited by the promotoras stated their fear of cancer "fatalism" negatively impacted screening for cervical cancer [9]. Overall, the results of the study conducted in Chiapas, Mexico revealed that trained CHWs providing educational and social support improved cervical cancer screenings for Hispanic women in rural Mexico [9].

Poverty plays a major role in a women's ability to access resources for screening and treatment of cervical cancer. Research findings suggest that Mexico and developing countries have not been successful in reducing the rate of cervical cancer because of their limited human and financial resources for cervical cancer programs [2]. Because of Mexico's limited resources in impoverished areas, alternate low cost methods of cervical cancer testing have been implemented. To ensure cervical cancer 


\section{Nursing \& Healthcare International Journal}

screening program success quality assurance measures need to be in place. Quality assurance measures require regular monitoring and evaluation of cervical cancer screening programs [10].

In addition to lowered socioeconomic status to access cervical cancer screening services, Hispanic women report other barriers to cervical cancer screening. For example, fear of embarrassment from exposing one's genitalia especially with a male healthcare provider when Pap/HPV testing is performed, language barriers between patients and healthcare providers, transportation issues, lack of child care for appointments, and perceived discrimination from health care personnel [11]. Lack of knowledge regarding Pap/HPV testing also appears to be a barrier to screening. Studies reveal that women are confused as to the importance of Pap testing. One study revealed that over half of participating women in the study incorrectly believed that Pap testing assessed only for sexually transmitted diseases or infections [12]. Additional studies revealed positive outcomes to overcome cervical cancer screening barriers by using trained CHWs to educate participants in Spanish with culturally appropriate educational materials and providing social support improved the number of participants receiving Pap testing [13]. For example, a study conducted on 162 Hispanic residents who were without Pap testing for three years from a health clinic setting received an educational intervention led by CHWs. The intervention consisted of Spanish language media with the use of CHWs providing culturally appropriate support and education. The results of this study revealed that $76.5 \%$ of the participants who attended the educational intervention by the CHWs received Pap testing [13].

One paralleled randomized-controlled trial of 600 Hispanic women aged 21-64 who were based out of Yakima Valley, Washington was performed to assess the efficacy of two culturally-appropriate tailored educational programs designed to increase cervical cancer screening. One educational program was with Spanish language educational videos alone on cervical cancer and the other with the promotora providing the education with culturally appropriate educational materials mostly videos in Spanish on cervical cancer screening. The findings of the study reflected that women had a higher rate of compliance with cervical cancer screening when receiving educational and social support from the patient navigator also known as a promotora [14].

The literature supports the importance of having trained CHWs who are familiar with the Hispanic culture in order to increase cervical cancer screening services
[15]. There are many strategies to help reduce the rate of cervical cancer including the use of lay health workers in combination with media materials [16]. Hispanic women today continue to have many barriers related to cervical cancer screening and continue to be negatively impacted by this devastating disease. Efforts to reduce the burden of disease on Hispanic women in the United States and specifically Texas has led to this study.

The nurse managed clinic in Texas were this study was performed in 2017 has a large population of high-risk Hispanic women for cervical cancer who are living in poverty and have low literacy levels. The advanced practice nurses (APNs) working in the clinic primarily teach patients about cervical cancer prevention and screening with verbal instructions mostly in English. There are no protocols in place that reveal what information the APNs are teaching to the patients on cervical cancer screening. There currently is only one Spanish interpreter at the clinic to assist the APNs to communicate with the Hispanic women for cervical cancer prevention and screening, and only one part-time APN who speaks Spanish. With the lack of cervical cancer screening resources in the community for the uninsured and undocumented Hispanic women residents of the United States seen at the clinic, this study was needed. In addition, the clinic has had many no show appointments for cervical cancer screening with Pap/HPV testing and an intervention was needed to improve the number of Hispanic women keeping their appointments for Pap/HPV testing. It is hypothesized that promotoras who are trained to teach high-risk Hispanic women on cervical cancer prevention and screening may increase appointment compliance rates at a nurse managed clinic.

The purpose of this study is to assess the effectiveness of a trained community promotora-led cervical cancer educational intervention on cervical cancer prevention and screening. In addition, this study is to determine whether an educational intervention will increase the rate of kept appointments for Pap/HPV testing in high-risk Hispanic women who are indigent patients from a nurse managed clinic.

\section{Methods}

Promotoras recruited for this study included Hispanic women who were familiar with the patient population selected for the study. The nurse managed clinic is operated by one full-time APN and several part-time APNs who are also nursing faculty affiliated with a nearby university/hospital system. Regular staff and APNs associated with the clinic verbalized interest in the study. The promotoras recruited for the study received 


\section{Nursing \& Healthcare International Journal}

extensive training on their roles in the study, concepts related to cervical cancer prevention and screening, and educational class issues such as patient privacy and how to engage class participants. Written materials on cervical cancer prevention, screening guidelines from the American Cancer Society (ACS), and related information from the National Cancer Institute (NCI) were provided to the promotoras in both Spanish and English. These materials were given to the promotoras several weeks before starting the educational classes so that they may review the literature and ask questions if needed to the principal investigator. The potential 50 subjects for the control and the intervention group for this study were identified by a retrospective chart review that followed Institutional Review Board (IRB) approval. Inclusion criteria for this study were based from the guidelines for cervical cancer screening as defined by the U.S. Preventative Services Task Force (USPSTF). This included Hispanic females aged 21-65 years; last Pap testing over one year; and no history of hysterectomy unless for cervical cancer. Also, women who were pregnant were excluded from the study since the nurse managed clinic referred pregnant women to other agencies for prenatal care. The initiation of the retrospective chart review and data collection began with reviewing the clinic appointment schedules from the current date and going back 4 months and identifying Hispanic females aged 2165 . Once the potential Hispanic females were identified by the appointment schedules their electronic medical records were reviewed for eligibility. Continuous records review was conducted until 50 subjects were obtained for both the control and intervention group. The records of Hispanic women who did not meet the eligibility criteria were not included in the study. Overall the chart review process required reviewing over 200 electronic medical records by the principal investigator alone. This was a time consuming process due to the inability of the electronic medical record system to capture the appropriate data. Those medical records reviewed in the control group received the usual standard of care by the APNs for cervical cancer screening and were not to receive the educational intervention by the trained community promotoras. The intervention group were to receive the one-hour educational class on cervical cancer prevention and screening led by the trained community promotoras. Following the educational class, participants were given the opportunity to schedule an appointment for Pap/HPV testing for a later date.

\section{Recruitment}

Following IRB approval, 50study participants were chosen for the control group and 50 participants were chosen for the intervention group via a retrospective electronic chart review. All study participants' age 21 to 65 years were assessed for eligibility for cervical cancer screening based on the United States Preventive Services Task Force (USPSTF) recommendations for cervical cancer screening [17]. The Hispanic female employees from the clinic who were eligible for cervical cancer screening were included in the study. The control group did not receive the educational class intervention led by the promotoras, but were given a verbal explanation (standard clinic procedure) regarding cervical cancer screening with Pap/HPV testing by an APN in the nurse managed clinic. Hispanic women eligible for cervical cancer screening were telephonically invited to participate in the study and attend the educational intervention. The telephone recruitment efforts conducted by the promotoras and with the oversight of the principal investigator lasted for several weeks. A IRB approved telephone script was created in English and Spanish to assist in the recruiting efforts by the promotoras.

\section{Intervention}

Promotoras offered four, one-hour cervical cancer prevention educational classes at various times and dates in order to accommodate the participants in the intervention group. Before implementing the educational classes, the promotoras were trained on how to conduct educational classes effectively. The role of the promotora was explained prior to the beginning of the study which included the goal and purpose of the study. Strict privacy and confidentiality were taught to the promotoras in regards to the participant releasing their personal health and demographic information that might be shared by the participant before, during, or after the educational class. Along with the principal investigator as an APN, the promotoras conducted the educational classes to the intervention group of Hispanic women in the study. The educational classes included the following information: general information about cervical cancer including statistics, signs and symptoms of cervical cancer, basic female anatomy, and risk factors for cervical cancer, screening schedule recommendations, and tests used for screening. All study participants in the intervention group received written Spanish and English educational materials from the American Cancer Society and National Cancer Institute to accompany the verbal teachings on cervical cancer prevention and screening. Educational classes occurred in an informal, quiet setting at the nurse managed clinic. Following the educational class, intervention group participants who attended the class were encouraged to schedule an appointment at the clinic for cervical cancer screening with Pap/HPV testing. 


\section{Nursing \& Healthcare International Journal}

\section{Results}

All the data collected from the electronic chart review which included the 50 control group participants and the 50 intervention group participants was decoded onto an Excel spreadsheet from a password protected personal computer. After reviewing the data, the 50 control group participants and 23 intervention group (those that attended the intervention) were then transferred to IBM SPSS Statistics for Windows, Version 23.0 for statistical analysis by performing a Chi Square and T-test. All of the comparisons used a probability of $<0.05$, which is considered statistically significant. Of the 50 eligible Hispanic women recruited for the intervention group, only 23 of them attended the educational intervention class. The remaining 27 eligible Hispanic women who did not attend the educational intervention were excluded from the statistical analysis results. The educational intervention led by the promotora with oversight from the principal investigator as APN was offered to 23 Hispanic women (attendance rate $=46 \%$ ) at the clinic to learn about cervical cancer prevention and screening. Data analysis with descriptive statistics, Chi Square test and T-test was used to analyze the total participants; differences of variables for both the control and intervention group excluding the outcome; and the outcome between the control group and intervention group that kept their appointments for cervical cancer screening with Pap/HPV testing are found in Tables 1-3, respectively.

Table 1 displays the total participant demographics sorted by mean age for the total participants, highest grade completed, last Pap test, history of cervical cancer, history of hysterectomy, risk factors, and HPV testing. The mean age for the total participants in the study was 41.73 and $20.5 \%$ had only a grade school education. The majority of the participants had received a Pap test in $2016(43.8 \%, \mathrm{n}=32)$. There were no women in the study that had a history of cervical cancer, and $(97.3 \%, \mathrm{n}=71)$ of the participants showed no history of a hysterectomy. The highest risk factor for cervical cancer in the participants was 3 or more pregnancies $(37.0 \%, n=27)$. There were participants that tested positive for HPV $(9.6 \%, \mathrm{n}=7)$, but $20.5 \%, \mathrm{n}=15$ of the participants who were required to have HPV testing did not receive testing. Overall there was a significant amount of missing data from the electronic medical records for most of the categories in Table 1.

\begin{tabular}{|c|c|}
\hline Age & $\begin{array}{c}\mathrm{n}(\%) \\
\text { Mean 41.73 } \\
\text { (Std. Deviation 10.767) }\end{array}$ \\
\hline \multicolumn{2}{|c|}{ Highest grade completed } \\
\hline Grade school & $15(20.5)$ \\
\hline High school & $7(9.6)$ \\
\hline Some college & $2(2.7)$ \\
\hline College graduate & $3(4.1)$ \\
\hline Total & $27(37.0)$ \\
\hline Missing data & $46(63.0)$ \\
\hline Total & $73(100.0)$ \\
\hline \multicolumn{2}{|c|}{ Last Pap test } \\
\hline Performed in 2016 & $32(43.8)$ \\
\hline Performed in 2015 & $27(37.0)$ \\
\hline $\begin{array}{c}\text { Performed in } 2014 \text { or } \\
\text { longer }\end{array}$ & $9(12.3)$ \\
\hline Total & $68(93.2)$ \\
\hline Missing data & $5(6.8)$ \\
\hline Total & $73(100.0)$ \\
\hline \multicolumn{2}{|c|}{ History of cervical cancer } \\
\hline No & $73(100.0)$ \\
\hline $\begin{array}{c}\text { History of } \\
\text { hysterectomy }\end{array}$ & \\
\hline Yes & $2(2.7)$ \\
\hline No & $71(97.3)$ \\
\hline Total & $73(100.0)$ \\
\hline \multicolumn{2}{|c|}{ Risk factors } \\
\hline None & $26(35.6)$ \\
\hline Tobacco use & $1(1.4)$ \\
\hline $\begin{array}{l}3 \text { or more } \\
\text { pregnancies }\end{array}$ & $27(37.0)$ \\
\hline Multiple partners & $1(1.4)$ \\
\hline Multiple risk factors & $6(8.2)$ \\
\hline Total & $61(83.6)$ \\
\hline Missing data & $12(16.4)$ \\
\hline Total & $73(100.0)$ \\
\hline \multicolumn{2}{|c|}{ HPV testing } \\
\hline Negative & $7(9.6)$ \\
\hline Positive & $2(2.7)$ \\
\hline Not required & $24(32.9)$ \\
\hline $\begin{array}{l}\text { Not performed but } \\
\text { required }\end{array}$ & $15(20.5)$ \\
\hline Total & $48(65.8)$ \\
\hline Missing data & $25(34.2)$ \\
\hline Total & $73(100.0)$ \\
\hline \multicolumn{2}{|c|}{ Appointments kept } \\
\hline Kept & $40(54.8)$ \\
\hline Not Kept & $33(45.2)$ \\
\hline Total & $73(100.0)$ \\
\hline
\end{tabular}

Table 1: Discriptives of Control Group $(\mathrm{N}=50)$ and Intervention Group ( $\mathrm{N}=23)$. 


\section{Nursing \& Healthcare International Journal}

Table 2 displays the differences between the variables for the control and intervention group using the ChiSquare test. Differences occurred for the groups with the mean ages of $38.22 \mathrm{n}=50$ for the control group participants, and a mean age of $49.35 n=23$ for the intervention group participants when using a T-test. There were no statistical significance for highest grade completed ( $\mathrm{p}$.104), history of cervical cancer and hysterectomy (p .534) but there were statistical differences with risk factors (p.036), HPV testing (p .000) and last Pap test ( $\mathrm{p} .000)$. The control group had more participants with no risk factors for cervical cancer $(50.0 \%, \mathrm{n}=21)$ compared with the intervention group $(26.3 \%, n=5)$. In addition, the intervention group had more participants with 3 or more pregnancies $(47.3 \%, n=$ 9) and multiple risk factors as compared with the control group having 3 or more pregnancies $(43 \%, n=18)$ and multiple risk factors $(2.4 \%, \mathrm{n}=1)$. The HPV testing results revealed that there were more participants in the control group $(21.2 \%, \mathrm{n}=7)$ that had negative tests results and did not require HPV testing $(73.0 \%, \mathrm{n}=24)$, whereas the intervention group participants had more $(93.3 \%, \mathrm{n}=14)$ participants who required HPV testing, but the HPV testing was not performed. The last Pap testing comparison with groups demonstrates that the intervention group had more $(50.0 \%, \mathrm{n}=9)$ participants who had a Pap test performed in 2014 or longer, compared with the control group (0\%). In addition, the intervention group had fewer participants $(11.0 \%, \mathrm{n}=2)$ than the control group $(50.0 \%, \mathrm{n}=50)$ who had a Pap test performed in 2015, and the intervention group had a fewer participants with Pap testing in $2016(39.0 \%, \mathrm{n}=7)$ than the control group $(50.0 \%, \mathrm{n}=25)$.

\begin{tabular}{|c|c|c|c|}
\hline & Control n (\%) & Intervention n (\%) & Total \\
\hline Agemean & 38.22 & 49.35 & \\
\hline \multicolumn{4}{|c|}{ Highest grade completed } \\
\hline grade school & $5(50.0)$ & $10(59.0)$ & 15 \\
\hline high school & $1(10.0)$ & $6(35.0)$ & 7 \\
\hline some college & $2(20.0)$ & $0(0.0)$ & 2 \\
\hline college graduate & $2(20.0)$ & $1(5.9)$ & 3 \\
\hline Total & 10 & 17 & 27 \\
\hline \multicolumn{4}{|c|}{ Last Pap test } \\
\hline performed in 2016 & $25(50.0)$ & $7(39.0)$ & 32 \\
\hline performed in 2015 & $25(50.0)$ & $2(11.0)$ & 27 \\
\hline performed in 2014 or longer & $0(0.0)$ & $9(50.0)$ & 9 \\
\hline Total & 50 & 18 & 68 \\
\hline \multicolumn{4}{|c|}{ Risk factors } \\
\hline none & $21(50.0)$ & $5(26.3)$ & 26 \\
\hline tobacco use & $1(2.4)$ & $0(0.0)$ & 1 \\
\hline 3 or more pregnancies & $18(43.0)$ & $9(47.3)$ & 27 \\
\hline multiple partners & $1(2.4)$ & $0(0.0)$ & 1 \\
\hline multiple risk factors & $1(2.4)$ & $5(10.2)$ & 6 \\
\hline Total & 42 & 19 & 61 \\
\hline
\end{tabular}

Table 2: Differences in Variables between Control Group ( $\mathrm{N}=50)$ and Intervention Group ( $\mathrm{N}=23$ ).

Table 3 displays the outcome for the study for the groups who kept their appointments and those Hispanic women who did not keep their appointments. The ChiSquare test results revealed that the control group had fewer $(52.0 \%, \mathrm{n}=26)$ participants who kept their appointment for cervical cancer screening with Pap/HPV testing compared with the intervention group $(60.0 \%, \mathrm{n}=$ 14). Overall, the Chi-Square test revealed a probability of .326 which indicates that there is no statistically significant difference in the appointments kept across the two groups.

\begin{tabular}{|c|c|c|c|}
\hline & Kept n (\%) & Not Kept n (\%) & Total \\
\hline Control & $26(52.0)$ & $24(48.0)$ & 50 \\
\hline Intervention & $14(60.0)$ & $9(39.0)$ & 23 \\
\hline Total & $40(55.0)$ & $33(45.0)$ & 73 \\
\hline
\end{tabular}

Table 3: Appointments Kept For Cervical Cancer Screening. 


\section{Nursing \& Healthcare International Journal}

\section{Discussion}

The goal of this study was to assess the effectiveness of utilizing trained community promotoras to teach highrisk Hispanic women about cervical cancer prevention and screening in order to increase the rate of kept appointments for Pap/HPV testing. Overall, all participants who attended the educational class scheduled an appointment for cervical cancer screening with Pap/HPV testing, but only 12 participants kept their appointments for Pap/HPV testing. The participants that attended the educational class displayed enthusiasm and a willingness to be screened for cervical cancer. For the women who did not keep their appointments for Pap/testing, the possibility of having a fear of cancer could have been the cause along with other factors due to the number of excuses given when follow-up telephone calls were made by the promotoras. The promotoras and APN demonstrated respect to the Hispanic women including making additional reminder telephone calls for the educational classes. However, this might have impacted the participants own self-motivation for attendance to the classes. The geographic location of the clinic for the educational classes appeared adequate, but the class times and dates may have impacted the attendance of those potential participants based on the excuses given by the Hispanic women for nonattendance when follow-up telephone calls were made. The educational intervention classes were conducted at various dates and times and had a total of 23 participants in attendance with $100 \%$ of them scheduling an appointment for Pap/HPV testing at the end of the class. In addition, the participants in the educational class specifically requested the APN/principal investigator in attendance during the educational class to perform the cervical cancer screening with Pap/HPV testing at the conclusion of the study. Forming a trusting relationship with the principal investigator/APN and promotora allowed the participants in the study to share some personal health information. For example, one participant in her late 50's reported a history of sexual abuse by her current partner.

\section{Limitations}

This study had several limitations. The first limitation was that the community promotoras full-time work obligations limited their availability to conduct the educational classes and recruitment for participants. The classes and telephone calls had to be made when the promotoras were available which was mostly in the early evenings or Saturdays. Secondly, even with reminder telephone calls by the promotoras to the participants' one to two days prior to the educational class events, there were participants who no-showed for the educational classes. The excuses provided by the participants for not attending the educational classes were as follows: "I am still at work and can't leave; I am babysitting my grandchildren; I have no transportation; I do not feel well; I forget the appointment". These responses reflect some of the additional barriers to screening that were not addressed during the implementation of this study. Further studies to increase cervical cancer screening in Hispanic women should include interventions to remove these barriers. For example, offering childcare and transportation services to attend the classes and the Pap/HPV testing appointments.

Another limitation for this study was the significant amount of missing patient health information from the electronic medical records pertaining to cervical cancer screening with Pap testing and HPV testing, risk factors, and highest educational level completed. In addition to missing data, a few of the potential participants who were telephoned for recruitment reported having a hysterectomy but the patients electronic medical records did not reflect this information. Also, some the potential participants reported having Pap testing elsewhere besides the nurse managed clinic were the study was performed, but it was not documented in their electronic medical record. These gaps in patient health information made it extremely challenging to determine who was eligible for participation in the study. Lastly, in order to obtain a significant sampling of participants for the study many hours were spent reviewing electronic medical records.

\section{Conclusion}

Further studies are needed to evaluate the effectiveness of using trained community promotoras for women at risk for other health issues besides cervical cancer and in other geographical settings. Offering educational classes and screening services with Pap/HPV testing in the community such as community centers, churches, and at the patients' place of employment and/or residency might improve attendance and kept appointments for screening. Recruiting promotoras that are not working full-time might be a better option to allow for increased flexibility of educational classes and recruitment efforts for study participants. Lastly, educating APNs and other providers on the need to document a complete health history in the patients' medical records would help increase the data collection efforts when conducting studies. 


\section{Nursing \& Healthcare International Journal}

\section{References}

1. World Health Organization (2013) Human papillomavirus (HPV) and cervical cancer.

2. Centers for Disease Control and Prevention (2014) HPV-Associated cervical cancer rates by race and ethnicity.

3. National Cancer Institute (2014) Cancer statistics factsheets: Cervix uteri cancer.

4. American Cancer Society (2014) Cervical cancer: Causes, risk, factors, and prevention topics.

5. Healthy People. gov (2014) Healthy People 2020.

6. Del Carmen MG, Avila Wallace M (2013) Effect of health care disparities on screening. Clin Obstet Gynecol 56(1): 65-75.

7. Partridge E, Fouad M (2010) Community-driven approaches for reducing health disparities in cancer. Jama 303(11): 1090-1091.

8. Ramirez AS (2014) Fatalism and cancer risk knowledge among a sample of highly acculturated Latinas. J Cancer Educ 29(1): 50-55.

9. Elliott PF, Belinson SE, Ottolenghi E, Smyth K, Belinson JL (2013) Community health workers, social support and cervical cancer screening among highrisk groups in rural mexico. J Health Care Poor Underserved 24(24): 1448-1459.

10. Ali F, Kuelker R, Wassie B (2012) Understanding cervical cancer in the context of developing countries.
Annals of Tropical Medicine and Public Health 5(1): 3-15.

11. Corcoran J, Crowley M (2014) Latinas' attitudes about cervical cancer prevention: a meta-synthesis. J Cult Divers 21(1): 15-21.

12. Hawkins NA, Benard VB, Greek A, Roland KB, Manninen D, et al. (2013) Patient knowledge and beliefs as barriers to extending cervical cancer screening intervals in federally qualified health centers. Prev Med 57(5): 641-645.

13. Thompson B, Vilchis H, Moran C, Copeland W, Holte S (2014) Increasing cervical cancer screening in the united states-mexico border region. J Rural Health 30(2): 196-205.

14. Duggan C, Coronado G, Martinez J, Byrd TL, Carosso E, et al. (2012) Cervical cancer screening and adherence to follow-up among hispanic women study protocol: A randomized controlled trial to increase the uptake of cervical cancer screening in hispanic women. Bmc Cancer 12: 170 .

15. Flores BE, Acton GJ (2013) Older hispanic women, health literacy, and cervical cancer screening. Clin Nurs Res 22(4): 402-415.

16. Fernandez ME, Savas LS, Lipizzi E, Smith JS, Vernon SW (2014). Cervical cancer control for hispanic women in texas: Strategies from research and practice. Gynecol Oncol 132(1): S26-S32.

17. US Preventative Services Task Force (2013) Final recommendation statement: cervical cancer: screening. 